Recherches amérindiennes au Québec

\title{
Politiques indigénistes au Brésil, au Canada et en Australie
} Les défis de la pratique anthropologique dans divers contextes

nationaux

\section{Anthropology and Indigenist Policies in Brazil, Canada, and Australia

\author{
The Challenges to Anthropological Practice in Different National \\ Contexts
}

\section{Las políticas indigenistas en Brasil, Canadá y Australia \\ Desafíos de la práctica antropológica en diversos contextos nacionales}

\section{Stephen Grant Baines}

Volume 44, numéro 2-3, 2014

Amérique latine, Guyane française, États-Unis, Canada, Nouvelle-Calédonie

URI : https://id.erudit.org/iderudit/1030962ar

DOI : https://doi.org/10.7202/1030962ar

Aller au sommaire du numéro

Éditeur(s)

Recherches amérindiennes au Québec

ISSN

0318-4137 (imprimé)

1923-5151 (numérique)

Découvrir la revue

Citer cet article

Baines, S. G. (2014). Politiques indigénistes au Brésil, au Canada et en Australie : les défis de la pratique anthropologique dans divers contextes nationaux. Recherches amérindiennes au Québec, 44(2-3), 5-12.

https://doi.org/10.7202/1030962ar
Résumé de l'article

Cet article examine certains des défis rencontrés par l'ethnologie autochtone au Brésil, au Canada et en Australie au cours des dernières décennies, en mettant l'accent sur les situations où se produit le contact interethnique entre peuples autochtones et États nationaux et dans lesquelles l'anthropologue intervient au moyen de recherches politiquement engagées. La représentation des peuples autochtones dans les trois pays s'est renforcée depuis la consolidation des mouvements politiques autochtones à partir des années 1970, au moment où les grandes sociétés minières, forestières, d'élevages, agro-industrielles et hydroélectriques convoitaient les ressources naturelles sur les territoires autochtones. L'anthropologue travaille dans des contextes fortement politisés en collaboration avec des agents sociaux des communautés autochtones et de la société nationale, tant du gouvernement que des entreprises actives sur les territoires autochtones. Un des rôles joués par l'anthropologue consiste à interpréter les situations complexes d'interventions gouvernementales et de l'indigénisme entrepreneurial en analysant et en contextualisant les différentes opinions des intervenants sociaux qui y prennent part. 


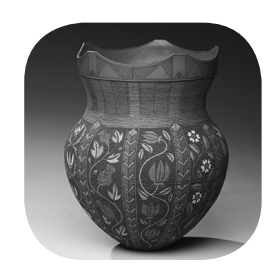

Politiques indigénistes au Brésil, au Canada

et en Australie

Les défis de la pratique anthropologique

dans divers contextes nationaux ${ }^{1}$

\section{Stephen Grant \\ Baines \\ Département \\ d'anthropologie, \\ ICS \\ Université \\ de Brasília, \\ Brasília, D.F. \\ Traduit du \\ portugais par \\ Alice Fiuza}

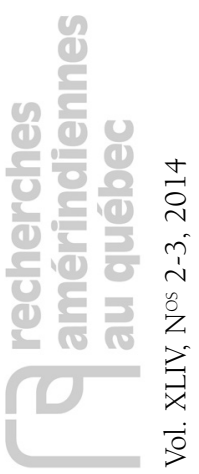

$\bigcup^{\mathrm{ET}}$ ARTICLE EXAMINE certains des défis que l'ethnologie autochtone (une branche de l'anthropologie sociale consacrée à l'étude des peuples autochtones) a rencontrés au Brésil, au Canada et en Australie au cours des dernières décennies, en mettant l'accent sur les situations où il y a un contact interethnique entre peuples autochtones et États nationaux et où l'anthropologue se trouve dans un contexte fortement politisé. Dans les trois pays, la représentation des peuples autochtones s'est renforcée depuis la consolidation des mouvements politiques autochtones à partir des années 1970, au moment où les grandes sociétés minières, forestières, d'élevages, agroindustrielles et hydroélectriques convoitaient les ressources naturelles sur les territoires autochtones.

Nous avons entamé une étude en ethnologie comparée en 1991, dans le cadre du projet de recherche sur «les styles d'anthropologies » coordonné par le professeur Roberto Cardoso de Oliveira, en examinant le style d'ethnologie autochtone pratiqué en Australie (Baines 1995). À partir de 1995, nous avons élargi la recherche pour inclure une étude sur le style d'ethnologie réalisé au Canada (Baines 1996). Nous avons utilisé, comme référence pour les recherches en ethnologie comparée, l'ethnologie autochtone pratiquée au Brésil, où nous avons obtenu un doctorat en anthropologie. Bien que ces trois pays aient des histoires de colonisation très différentes et des politiques autochtones diverses, il existe beaucoup de similarités dans les processus globaux qui touchent actuellement les peuples autochtones. Au cours du présent texte, nous aborderons certains des défis concernant la participation d'anthropologues dans des contextes d'intervention sociale d'États nationaux, des défis qui se sont accrus au cours des trois dernières décennies, contribuant fréquemment à l'expansion et au renforcement de l'ethnologie autochtone tant au Brésil que dans d'autres pays de colonisation européenne où les États se sont étendus sur les territoires traditionnels ${ }^{2}$ des peuples autochtones. Cette participation entraîne la formulation de nouvelles questions et problématiques en anthropologie.

Les conventions internationales, comme la Convention 169 de l'OIT (Organisation internationale du travail) de $1989^{3}$ et la Déclaration des Nations unies sur les droits des peuples autochtones de $2007^{4}$, ont souligné le besoin de respecter et de protéger les manifestations culturelles des populations natives. Dans chaque État national, les législations et les politiques indigénistes ont eu à considérer les demandes des mouvements autochtones. La présence d'agences internationales de coopération et de développement a également contribué au renforcement de la représentation autochtone. Les multiples rôles que jouent les anthropologues 
dans les programmes ou organismes gouvernementaux, organisations non gouvernementales, programmes de coopération internationale ou autres entreprises, les mettent dans de nouvelles situations où ils font face à des défis et des dilemmes. Le rôle de l'anthropologue a subi des transformations : ce professionnel n'agit plus en tant qu'intermédiaire des peuples autochtones auprès du gouvernement (Ramos 1998), comme c'était le cas dans les années 1960 et 1970 quand il y avait peu de porte-parole autochtones qualifiés. Aujourd'hui, il agit plutôt comme expert-conseil auprès des peuples autochtones qui gèrent leurs propres projets (Oliveira et Iglesias 2002). Dans la dernière décennie, il y a eu un effort à l'échelle internationale, de la part des peuples autochtones, pour professionnaliser les cadres de leurs organisations et de leurs leaders (Luciano 2006). Bien que, par rapport aux peuples autochtones, le rôle de l'anthropologue ait beaucoup changé en ce qui a trait à leur représentation, il y a aussi des fonctions importantes exercées par des anthropologues au sein d'organisations non gouvernementales de soutien aux peuples autochtones, notamment comme conseillers de projets, mais aussi au sein d'organismes gouvernementaux et comme consultants pour des entreprises. Au Brésil, avec l'imposition de grands projets de développement en Amazonie, comme, entre autres, les usines hydroélectriques (UHE) Belo Monte, Balbina, Tucuruí, l'activité minière industrielle, l'agriculture et l'élevage des grandes entreprises, l'anthropologue fait face à des défis de plus en plus importants en traitant des situations extrêmement complexes où les pressions économiques des grandes entreprises exercent une forte influence sur les gouvernements fédéraux, régionaux et locaux, ainsi que sur les dirigeants autochtones. Or, ceuxci se voient de plus en plus obligés de négocier directement avec les entreprises (Ritter 2009), en ayant peu ou aucun droit de veto effectif relativement à l'implantation des projets de développement sur leurs territoires. Dans certains cas, comme pour le Programme Waimiri-Atroari (Baines 1993) et le Programme Parakanã, de l'Eletronorte, ainsi que le Programme Avá-Canoeiro de la société Furnas (Silva 2005), les entreprises exploitant dans les territoires autochtones remplissent le rôle du gouvernement fédéral en commanditant des programmes d'aide indigénistes. Ces programmes sont généralement liés aux intérêts de l'entreprise, sous la forme d'un indigénisme entrepreneurial visant à mitiger les impacts néfastes de ces grands chantiers, tout en laissant croire que les projets de développement peuvent être convertis en bénéfices pour les peuples autochtones (Baines 1993).

Ritter (2009) présente une situation semblable d'application de la législation concernant les droits aux territoires autochtones en Australie, en réfléchissant sur le processus actuel qui consiste à établir des accords dans le cadre du Native Title Act de 1993. Lauteur met l'accent sur les accords établis entre le secteur minier et les peuples aborigènes lorsqu'il y a des ressources minières sur leurs territoires. À partir de sa propre expérience à titre de consultant dans les négociations entre les aborigènes et les entreprises, Ritter analyse des cas empiriques et brise certains mythes forgés autour du concept du Native Title et qui ont tendance à le représenter comme étant une disposition autochtone ou marginale. Dans le même ouvrage, Ritter définit le processus de création d'accords entre les compagnies minières et les peuples aborigènes comme un "marché » où le droit de négocier les processus fonctionne comme un genre de « centre commercial virtuel » dans lequel les compagnies minières achètent leurs autorisations pour réaliser des recherches et l'exploitation de minerais chez les peuples autochtones qui agissent comme commerçants en vendant leur consentement aux entreprises. Lauteur ajoute que, dans certains cas, des porteparole autochtones deviennent des hommes d'affaires.

Nous avons examiné certains des défis que rencontre l'anthropologue travaillant en situation de contact interethnique avec les peuples autochtones dans ces trois pays au cours des dernières décennies du Xx ${ }^{\mathrm{e}}$ siècle. Durant les années de la dictature militaire au Brésil (1964 à 1985), il y avait consensus au sein de la majorité des anthropologues pour défendre les droits amérindiens contre les politiques de développement. La question autochtone servait de prétexte pour soulever des critiques envers le gouvernement (Ramos 1998). Il y avait une unanimité pour partager un accord avec les Amérindiens, créant avec les peuples autochtones un style d'anthropologie qui mettrait en valeur l'engagement politique de l'anthropologue, caractérisant ainsi le style d'ethnologie qui se produisait au Brésil (Peirano 1991; Ramos 1990).

Dans les années 1960 et 1970, au Canada et en Australie, nombre d'anthropologues se sont associés à des idéaux socialistes de la gauche politique, et quelques-uns se sont joints au mouvement politique autochtone. D'après un anthropologue québécois faisant référence à la décennie 1970, «[c]elle-là fut une espèce d'âge d'or de la militance $[\ldots]$ quand régnait l'idée qu'il y avait des solutions faciles pour presque tous les problèmes sociaux » (Baines 1996: 21), et quand le mouvement autochtone présentait « un discours très radical. » "Ce discours ressemblait beaucoup au discours nationaliste des Québécois radicaux ». (ibid. : 21) Selon ce même anthropologue, pendant cette décennie-là, il était courant au Québec de voir des politiciens nationalistes québécois partager la scène avec des dirigeants autochtones, une façon de s'identifier avec les Amérindiens pour ce qui était vu comme une lutte commune contre le colonialisme des Canadiens anglophones. Toutefois, les prétentions séparatistes de certains politiciens québécois ont vite déçu la plupart des dirigeants amérindiens qui craignaient un traitement encore pire de la part d'un Québec indépendant que celui qu'ils recevaient du gouvernement fédéral canadien.

En Australie, les partis politiques de gauche, notamment l'Australian Labour Party, appuyaient le mouvement politique aborigène, ce qui a mené au référendum national de 1967 pour la reconnaissance des droits absolus des peuples aborigènes australiens à la citoyenneté, et à l'Aboriginal Land Rights (Northern Territory) Act de 1976, 
formulé par le gouvernement du Parti travailliste et approuvé par le gouvernement conservateur qui lui a succédé.

Après plus de trente ans, certains anthropologues ont commencé à mettre en question les politiques autochtones du moment, fondées sur des idéaux socialistes soulignant les valeurs anticolonialistes et défendant l'autodétermination autochtone, alors qu'à cette époque on croyait, naïvement, qu'en rendant les droits territoriaux aux peuples natifs, on pourrait faire marche arrière dans l'histoire du colonialisme et les ramener ainsi dans un passé où les sociétés fonctionneraient comme on suppose qu'elles le faisaient avant les décennies de destruction culturelle, de décimation de population, de déplacements forcés et autres violences de l'histoire coloniale. À la suite de l'application de ces politiques, on a observé dans quelques communautés autochtones des situations d'extrême violence, des taux de suicide beaucoup plus élevés que chez les non-autochtones, des taux importants d'alcoolisme et d'usage d'autres substances, des violences domestiques et sexuelles, ainsi que des accusations de détournement de fonds au sein des administrations autochtones. Dans les années 1990, Noel Dyck (1993) a signalé le dilemme devant lequel se trouvaient les anthropologues qui travaillaient avec des peuples amérindiens au Canada: choisir de se taire à propos des aspects tragiques de ces sociétés qui auraient renforcé les préjugés racistes concernant « l'autochtone », ce qui aurait été perçu comme une attitude condescendante devant des situations tragiques, ou choisir d'exposer les réalités, souvent tristes, de la vie quotidienne des communautés autochtones, au risque d'attirer l'attention du public sur des aspects qui pourraient être utilisés contre les Amérindiens par leurs traditionnels ennemis souhaitant s'emparer de leurs territoires. Largument de Dyck (1993) était qu'il est nécessaire que l'anthropologue affronte ces situations et qu'il prenne en considération les différentes réponses autochtones, conférant ainsi une voix plus forte aux Amérindiens dans ses publications.

Les multiples rôles que l'anthropologue joue dans les programmes ou les organismes gouvernementaux, dans les organisations non gouvernementales, dans les programmes de coopération internationale ou d'autres entreprises, le mettent dans des situations nouvelles où il est confronté à des défis, puisqu'il s'agit de conjonctures où :

\begin{abstract}
Les peuples autochtones se trouvent placés dans des relations sociales tout à fait inégales vis-à-vis des segments de la société nationale/mondiale, et ils méritent une attention spéciale de la part des anthropologues en raison de leur vulnérabilité devant les pressions économiques et politiques des grandes entreprises qui ont le pouvoir d'influencer les décisions des gouvernements d'États nationaux dans des situations de contacts interethniques fortement politisés. (Baines 2004 : 30)
\end{abstract}

Dans les contextes d'intervention sociale, l'anthropologue travaille avec de multiples agents sociaux comme des dirigeants autochtones d'échelle nationale, régionale et locale, des intervenants juridiques, des agents d'organisations gouvernementales, non gouvernementales et de nombreux autres acteurs. Oliveira (1998) s'est penché sur les dangers et les pièges qui peuvent naître de la collaboration entre anthropologues et avocats, car, selon lui, «cette association rassemblant trois éléments - la recherche anthropologique, l'action juridique et les demandes autochtones - n'apportera pas toujours des solutions heureuses aux trois paliers » (Oliveira 1998: 269). Dans le contexte canadien, James Waldram (1993) a remis en question les limites de l'activisme des anthropologues dans des situations où il est question de grands projets hydroélectriques dans des territoires amérindiens, sujet déjà débattu par Robert Paine dans les années 1980 (Paine 1985).

Les situations d'intervention sociale sont multiples: des situations où l'anthropologue agit à titre de conseiller d'organisations autochtones, où il travaille pour des organisations gouvernementales et non gouvernementales, pour des sociétés minières ou hydroélectriques fonctionnant à l'intérieur des territoires autochtones. Les situations sont très complexes, comme dans le cas d'interventions gouvernementales avec imposition de politiques autochtones polémiques et contradictoires qui polarisent souvent l'opinion des anthropologues expérimentés.

\section{L' " INTERVENTION FÉDÉRALE " DE 2007 DANS Le Territoire dU Nord, en AUSTRAlie}

Nous avons effectué une brève analyse de la juxtaposition entre l'anthropologie et l'indigénisme $e^{5}$ dans le cas d'une intervention sociale sans précédent du gouvernement fédéral d'Australie, en 2007, dans le Territoire du Nord (Northern Territory - NT) ${ }^{6}$, qui a eu des conséquences importantes pour les peuples aborigènes d'Australie. Il s'agit d'une intervention sociale gouvernementale qui a divisé beaucoup d'anthropologues réalisant des recherches dans ce pays auprès des communautés autochtones et participant à titre de conseillers ou de consultants.

Lintervention fédérale de 2007 a eu lieu en réponse à un rapport intitulé Little Children are Sacred (Les petits enfants sont sacrés), produit par une commission d'enquête sur la protection des enfants aborigènes contre les agressions sexuelles. Cette commission, réclamée par le gouvernement du Territoire du Nord pour enquêter sur les dénonciations de violences sexuelles, publia son rapport le 15 juin 2007. Il établissait que l'agression sexuelle des enfants en communautés aborigènes du NT avait atteint des niveaux critiques et il exigeait que ces mauvais traitements soient "considérés comme une urgente question d'importance nationale tant par les gouvernements du Territoire du Nord que par le gouvernement fédéral » (Northern Territory Board of Inquiry... 2007 : 9).

La parution de ce rapport a poussé le gouvernement fédéral à formuler la Northern Territory National Emergency Response, publiée le 21 juin 2007. Dans ce document, le gouvernement australien annonçait l'interdiction de consommer des boissons alcoolisées dans les communautés aborigènes du Territoire du Nord, et l'imposition d'un contrôle sur le pourcentage des allocations sociales versées aux aborigènes les obligeait à s'en servir exclusivement pour l'achat de biens de première nécessité tels que définis par le gouvernement fédéral. Le document 
ordonnait également le déploiement d'un contingent spécial de policiers et d'agents de la santé publique dans la région et suspendait le système d'autorisations donnant accès aux communautés aborigènes. En 2010, James Anaya, des Nations unies, a jugé cette réponse d'urgence du gouvernement comme discriminatoire d'un point de vue racial, en plus de violer les droits civils des peuples aborigènes dans le Territoire du Nord. M. Anaya reconnaissait le besoin de prendre des mesures urgentes, mais il soulignait que des actions comme l'interdiction d'accès à l'alcool et à la pornographie ainsi que l'imposition d'un contrôle sur les allocations sociales pour assurer l'achat de biens de nécessité représentaient une restriction à l'autonomie individuelle.

Lintervention fédérale de 2007 a produit une division entre les anthropologues qui travaillaient auprès des aborigènes en Australie: d'une part, il y avait ceux qui défendaient l'intervention gouvernementale, faisant valoir qu'en cas de situations critiques de violence dans les communautés aborigènes, le gouvernement ne pouvait rester indifférent; d'autre part, il y avait ceux qui, s'appuyant sur des arguments fondés sur le respect aux droits de la personne, arguaient que l'autonomie politique aborigène devait prévaloir. Entre ces deux extrêmes, quelques anthropologues australiens cherchaient un compromis, défendant certains types d'interventions gouvernementales dans des cas précis, mais non de la manière imposée par l'intervention fédérale de 2007.

Ladite intervention fut perçue par de nombreux anthropologues et dirigeants aborigènes comme un important recul des progrès du mouvement autochtone dans le pays. Depuis la croissance du mouvement politique autochtone tant au Brésil et en Australie qu'au Canada à partir des années 1970, et surtout après la Constitution de 1988 au Brésil, les organisations autochtones prennent désormais du poids, et la représentation autochtone caractérise les politiques autochtones.

La déclaration d'une « urgence nationale » dans le Territoire du Nord de l'Australie le 21 juin 2007 par le Premier ministre australien de l'époque, John Howard, et le ministre des Affaires indigènes, Mal Brough, relativement aux allégations d'agressions sexuelles contre des enfants aborigènes du NT, a été suivie de mesures sans précédent dans la politique autochtone australienne, relatées plus haut, par lesquelles le gouvernement exerça un contrôle direct sur les communautés aborigènes, outrepassant l'autorité du gouvernement du Territoire du Nord et celle des organisations communautaires locales, sous prétexte d'imposer un environnement sain et sécuritaire aux enfants aborigènes.

Parmi les anthropologues qui ont soulevé de véhémentes critiques contre l'intervention fédérale dans les vies de plus de 40000 aborigènes au NT, Jon Altman et Melinda Hinkson (2007) de l'Australian National University (ANU) ont publié, en signe de protestation, une série d'articles écrits par des dirigeants aborigènes et des universitaires. Ces articles décochaient des critiques envers cette intervention draconienne, selon les perspectives des droits de la personne, des politiques de santé et de la lutte contre l'alcoolisme, des réformes du système de protection sociale et des droits territoriaux, de la représentation et de la réconciliation aborigènes, ainsi que de la reconnaissance de la diversité culturelle. Les articles partageaient un consensus selon lequel toute intervention gouvernementale ne peut avoir de résultats positifs que si elle comprend les objectifs et les aspirations des peuples autochtones, en leur concédant le droit de prendre directement part aux processus visant à affronter les problèmes sociaux et à projeter des avenirs durables pour leurs communautés.

Selon Hinkson, les mesures restrictives de l'État pour imposer le contrôle « constituent une intervention gouvernementale sans précédent face à la politique aborigène dans les derniers quarante ans » (ibid. : 1). Altman remarque : "Ce plan radical visant à transformer des sociétés organisées selon des principes d'affinité en des sociétés organisées autour du marché est fondé sur des notions hautement discutables [...] » (ibid. : 307). Les articles suggéraient que les objectifs réels du gouvernement étaient de profiter de ce rapport sur l'agression sexuelle envers les enfants pour imposer aux peuples aborigènes des politiques d'intégration accélérée, une tentative de forcer ceux-ci à entrer dans l'économie capitaliste de marché.

Merlan (2010) constatait qu'il y avait eu de nombreuses réactions à l'intervention de 2007, et il mentionnait deux réponses diamétralement opposées ayant comme principe le statut de l'agression sexuelle des enfants. Une des perspectives présentées arguait qu'en réalité la question de l'agression sexuelle d'enfants avait été l'exploitation opportuniste d'un sujet délicat. Certains auteurs ont suggéré qu'il s'agissait d'un prétexte utilisé par le gouvernement fédéral pour reprendre le contrôle sur les territoires et les communautés aborigènes, tandis que d'autres, d'une manière plus générale, ont déclaré qu'il s'agissait d'une accusation motivée par des intérêts politiques. Toutefois, ajoute Merlan, ces interprétations de l'intervention, qui cherchaient des prétextes occultes, ignoraient souvent l'accent mis dans le rapport sur le fait que les agressions sexuelles contre les enfants avaient été sous-estimées. Lautre type de réaction allait dans le sens que l'intervention tenait compte d'un problème réel, même si bon nombre de ceux qui ont souscrit à ce point de vue doutaient de l'efficacité de la manière dont elle avait été appliquée. Merlan soulève également que les auteurs qui admettaient l'existence de problèmes graves exigeant de l'attention disaient aussi être conscients de la complexité des questions humaines qui étaient en cause et des limitations de tout type d'« intervention » pour les régler.

Une autre publication récente qui a soulevé de fortes réactions parmi les anthropologues travaillant auprès des aborigènes en Australie est la série d'essais très polémiques de l'anthropologue-linguiste Sutton (2009), de l'Université de Melbourne, intitulée The Politics of Suffering: Indigenous Australia and the end of the liberal consensus, qui s'avère être une réflexion plus élaborée d'un article du même auteur 
publié huit ans auparavant (Sutton 2001). Dans ce livre, Sutton annonce qu'il rompt le silence de certains de ses collègues anthropologues qui, alignés avec la gauche politique depuis les années 1970, appuient le mouvement visant à la décolonisation des peuples aborigènes de l'Australie. L'auteur fonde ses arguments sur son expérience personnelle : une recherche sur le terrain dans les années 1970, dans la péninsule du cap York au nord de l'État du Queensland, auprès du peuple wik de Aurukun, une participation postérieure à des projets d'aide communautaires, et son rôle à titre de chercheur principal dans le processus de revendication territoriale du peuple wik. Sutton défend son soutien aux interventions gouvernementales en prétextant qu'il est impossible pour un anthropologue de rester silencieux devant les situations tragiques dans lesquelles se trouvent de nombreuses communautés aborigènes. Il convient ici de mentionner que le peuple qui était le sujet de la recherche de Sutton ne se trouve pas dans le Territoire du Nord, lieu de l'intervention fédérale de 2007.

Ce même auteur ajoute que des mesures ont été nécessaires pour empêcher ces communautés de chuter vers l'effondrement total (descent into dysfunction) [Sutton 2009 : 3]. Il commence son livre en racontant qu'au cours d'une visite à Aurukun en l'an 2000 pour prendre part aux funérailles de deux amies aborigènes, il a observé que ce village

était passé d'une communauté autrefois viable et vibrante, telle [qu'il] l'avai[t] connue, à un territoire de tragédies. Les conflits violents, les viols, les attaques envers des enfants et des vieillards, ainsi que la négligence, avaient atteint des niveaux alarmants à partir de 1985 à la suite du libre accès aux boissons alcoolisées (Sutton 2009 : 1).

Lauteur blâme des collègues anthropologues d'être restés silencieux. Il cherche également des éléments de la vie traditionnelle aborigène pouvant expliquer la situation de violence actuelle et il se porte à la défense de l'intervention gouvernementale.

Ce livre a provoqué des critiques et a accru la division entre les anthropologues et les dirigeants aborigènes fortement opposés à l'intervention fédérale de 2007 et d'autres qui, comme Sutton, appuient l'intervention comme une mesure nécessaire pour changer les conditions tragiques dans lesquelles se trouvent quelques communautés autochtones. Pour leur part, d'autres anthropologues et quelques ethnologues soutiennent une certaine forme d'intervention gouvernementale, mais non sans fermement critiquer la manière dont le gouvernement fédéral a agi en 2007. Povinelli avance que The Politics of Suffering a été écrit dans l'intention de provoquer de vives réactions et d'attirer l'attention des médias (Povinelli 2010 : 18).

Pour mettre en contexte l'intervention fédérale de 2007 dans l'histoire des politiques indigénistes du gouvernement australien au cours des cinquante dernières années, il faut mentionner qu'en 1962, la législation fédérale a concédé le droit de vote aux peuples aborigènes lors des élections fédérales et, qu'en 1967, un référendum réalisé par le gouvernement fédéral a reconnu la citoyenneté absolue des peuples aborigènes d'Australie et des îles du détroit de Torrès (les deux grands regroupements aborigènes du continent australien) [Beckett 1988]. Au début des années 1970, un mouvement en faveur de la reconnaissance des droits territoriaux autochtones dans le Territoire du Nord est né, de pair avec le gouvernement travailliste (Australian Labour Party [ALP]) du Premier ministre de l'époque, Gough Whitlam. La loi fédérale adoptée par le gouvernement suivant était l'Aboriginal Land Rights (Northern Territory) Act de 1976. Cette loi a mis en place des mécanismes permettant aux peuples aborigènes d'amorcer des revendications territoriales fondées sur l'occupation traditionnelle des terres du NT. Devant l'impossibilité des peuples colonisés d'atteindre une autonomie complète, on a vite commencé à parler d'« autogestion autochtone » à l'intérieur de la structure de l'État national australien. En 1992, la Cour suprême d'Australie a rendu la décision Mabo, qui annulait le concept juridique de terra nullius et qui reconnaissait, pour la première fois de l'histoire coloniale en Australie, la possession traditionnelle autochtone de la terre. En 1999, le Parlement australien a approuvé la Motion de réconciliation, qui admettait l'histoire des maltraitances institutionnelles envers les peuples aborigènes du pays.

Vers la fin des années 1960 et durant les années 1970, de nombreux politiciens de gauche se sont joints à des anthropologues activistes pour appuyer le mouvement qui a vu le jour pendant la vague de décolonisation qui s'est répandue partout dans le monde. Les peuples aborigènes d'Australie ont eu accès au Welfare State (État-providence) qui leur accordait le droit à des allocations de chômage et de protection sociale, ainsi qu'un libre accès à la consommation de boissons alcoolisées. De même, des politiques visant à assurer leur autonomie ont été adoptées. Entre les décennies 1970 et 2000, certaines sociétés aborigènes d'Australie ont répondu à ces mesures par une consolidation accrue de leur autonomie. Cependant, d'autres communautés autochtones ont connu une croissance des taux d'alcoolisme, d'homicides, d'abus de substances toxiques, de violences domestiques et sexuelles contre des femmes, des enfants et des vieillards, ce que Sutton appelle social dysfonction (effondrement social).

En l'an 2000, au cours d'une réunion d'anthropologues en Australie, Sutton s'est vidé le cœur à l'endroit de certains d'entre eux qui appuyaient le mouvement visant la décolonisation des peuples aborigènes et il a défendu les interventions gouvernementales, en justifiant qu'il n'était pas possible de maintenir le silence devant ces situations tragiques et en exigeant des mesures pour sauver ces communautés aborigènes de l'effondrement social.

Sutton ajoute que « selon son expérience, Aurukun n'est pas la seule communauté à avoir sombré dans une situation d'effondrement total (dysfunction) durant les dernières décennies» (2009: 3). Lauteur affirme qu'après son retour de la communauté d'Aurukun en 2000, il est resté un an sans prendre part aux questions politiques internes des communautés, respectant l'autonomie autochtone. 
Toutefois, les situations tragiques l'ont conduit à abandonner l'argument que les non-autochtones ne devraient pas intervenir dans les questions concernant le récent et rapide effondrement social. Lauteur ajoute qu'en 2000 il a senti le besoin de se prononcer sur l'échec massif des politiques indigénistes gouvernementales des dernières décennies.

Sutton conclut son livre en traitant de la réconciliation bureaucratisée entre Australiens aborigènes et non aborigènes et il met en question l'accroissement de la racialisation de la société australienne, « quand on constate, écrit-il, un taux majoritaire de mariage entre aborigènes et non aborigènes (71,5 \% en 2006)» (2009: 212).

Malgré les échecs signalés plus haut, Sutton fonde son argumentation sur sa longue expérience à titre d'anthropologue et d'indigéniste politiquement engagé, et il présente un défi important lorsqu'il parle de la crise dans de nombreuses communautés autochtones, remettant en question le rôle de l'anthropologue dans de telles situations. Cependant, Sutton omet de mentionner que de nombreux problèmes soulignés dans son livre sont abordés dans les recherches d'autres anthropologues.

Nonobstant ses critiques envers les anthropologues, l'auteur propose peu de recommandations pour la politique indigéniste, compte tenu de ce qu'on peut attendre d'un anthropologue engagé politiquement, selon sa propre définition. Tout en condamnant vivement les politiques autochtones visant à promouvoir l'autodétermination et en reconnaissant les échecs des politiques autochtones autoritaires du passé, Sutton présente une perspective pessimiste et défend des stratégies d'intervention peu distinctes des politiques paternalistes du passé colonial. En exagérant l'influence des aspects internes de la culture autochtone pour expliquer l'effondrement social - considérations favorisées par les secteurs anti-autochtones d'Australie - et en polarisant la recherche d'arguments culturels et la validation d'explications simplistes concernant l'échec de la politique indigène, l'auteur ne tient pas compte de la complexité des contacts interethniques. Après avoir créé de grandes attentes au début de son ouvrage, en ce sens qu'il semblait vouloir affronter les situations tragiques d'une manière profonde, il laisse le lecteur déçu par ses conclusions partielles et mal conçues.

Parmi plusieurs anthropologues qui ont commenté l'intervention fédérale de 2007, Peterson (2010) a noté que le silence de nombreux anthropologues sur la violence dans les communautés autochtones peut s'expliquer, autrement que par la complexité des questions et la grande diversité régionale de l'Australie, par le fait que la formulation de politiques efficaces d'intervention sociale pour résoudre les problèmes sociaux actuels dans les régions isolées du continent exige que les limites du rôle normal de l'État - intervenir dans la vie privée - soient dépassées. Selon lui, il faudrait élaborer des politiques qui enfreindraient les droits de la vie privée et qui transformeraient les pratiques quotidiennes, les valeurs et les croyances, en intervenant dans un espace sociojuridique et comportemental jusqu'alors inaccessible, puisque protégé par la citoyenneté et d'autres droits, ce qui empêche l'avènement de changements dans ce qu'on définit comme la culture. Peterson soutient que, pour créer des politiques d'intervention sociale efficaces dans la vie des peuples et des communautés autochtones éloignées d'Australie, il est nécessaire de prendre des mesures qui outrepassent le rôle normal de l'État dans la vie de ses citoyens, car, selon lui, certains changements dans la tradition culturelle des aborigènes sont nécessaires.

\section{CONSIDÉRATIONS FINALES}

L'examen de ces exemples de travaux révèle comment des situations d'intervention concernant les relations sociales entre l'État national et les peuples autochtones peuvent entraîner une division d'opinions chez les anthropologues, les amenant à réévaluer leurs orientations théoriques devant de nouveaux défis. En tant que citoyens des États nationaux (Kapferer 1989), ils se retrouvent coincés dans une configuration complexe de loyautés nationales, impériales, régionales et ethniques omniprésentes dans leurs perspectives.

Dans une situation d'intervention sociale, au Brésil, au Canada ou en Australie, une étape importante pour l'anthropologue consiste à surmonter les limites de sa propre vision et à tenter d'ouvrir l'espace aux voix autochtones, afin de créer des actions pratiques ainsi que des textes collaboratifs et participatifs qui intègrent des relations dialogiques entre les autres acteurs sociaux et les autochtones, et où les opinions variées de ceux-ci sont prises en considération comme ayant la même importance que celles de l'anthropologue et des autres acteurs sociaux dans des situations complexes de contact interethnique.

Traitant des peuples aborigènes dans les régions plus isolées du nord et du centre du continent australien, lorsqu'elle parle de la politique qui prévaut derrière le débat concernant ces communautés, Austin-Broos (2010) met l'accent sur les questions de différences culturelles et d'inégalités. L'auteure analyse les raisons pour lesquelles les deux gouvernements, conservateurs et de gauche, ont échoué dans leurs politiques indigénistes à l'endroit des communautés isolées, et elle fait valoir qu'aussi longtemps que les indigénistes et les chercheurs ne tiendront pas compte des questions de différences culturelles et d'inégalités, l'énorme fossé entre les conditions de vie de ces communautés autochtones et celles du reste de la population australienne ne se réduira pas. Austin-Broos fournit un aperçu des opinions contradictoires des indigénistes au sujet de ces communautés aborigènes, des décennies de politiques indigénistes et des recherches qui ont échoué à évaluer la situation. Lauteure propose qu'il y ait, de la part du gouvernement australien, à la fois une reconnaissance des droits territoriaux autochtones et la création de perspectives d'emploi afin d'inverser la situation de désespoir dans laquelle se retrouvent ces communautés.

En ce qui concerne les limites de l'anthropologie pour influencer les décisions de l'État national, Hinkson (2010) affirme que les médias et le gouvernement continueront de 
choisir des bribes d'opinions provenant des anthropologues - comme divers autres types de commentaires afin de valider leurs propres lignes d'action. Pour que l'analyse anthropologique puisse avoir une influence sur le discours public, les anthropologues devront trouver de nouvelles manières de communiquer les résultats de leurs recherches. Dialectiquement, cela dépendra de l'intérêt de la société, au sens large, à entendre ce que les anthropologues ont à dire. En fait, cela proviendra d'une reconfiguration de la place de la recherche universitaire dans la sphère publique.

Les cas discutés ci-dessus rendent compte des diverses réponses, parfois conflictuelles, des anthropologues devant ces défis. La situation actuelle d'expansion capitaliste sans précédent, partout dans le monde, et la réduction des ressources octroyées par les gouvernements aux programmes sociaux présentent un nouveau scénario aux peuples autochtones. Ceux-ci vivent de plus en plus de situations où ils doivent négocier directement, et dans des termes tout à fait inégaux, avec des gigantesques consortiums d'entreprises qui ont des exploitations sur leurs territoires. Il revient à l'anthropologue soucieux des droits autochtones d'accompagner ces différents processus d'intervention, mettant en pratique une anthropologie politiquement engagée afin d'analyser et de mettre en lumière les stratégies permettant de relever ces nouveaux défis.

\section{Notes}

1. Document préparé pour le Groupe de travail (GT 55) "Politique interethnique autochtone en perspective comparée », organisé par Cristhian Téofilo da Silva et Martin Hébert, au cours de la $28^{\mathrm{e}}$ Réunion brésilienne d'anthropologie, tenue du 2 au 5 juillet 2012 à São Paulo, Brésil. Mes remerciements aux coordonnateurs pour avoir accepté ce travail.

2. Nous nous alignons sur la pensée de Gallois (2004 : 39) selon laquelle « la différence entre "terre" et "territoire" fait référence à différents points de vue et acteurs engagés dans le processus de reconnaissance et de démarcation d'une Terre autochtone. La notion de "Terre autochtone" fait référence au processus politico-juridique mené sous l'égide de l'État, tandis que celle de "Territoire" se rapporte à la construction et à l'expérience, variable selon la culture, de la relation entre une société particulière et sa base territoriale ». Le concept juridique de « Terre autochtone » fait référence au processus de régularisation des territoires des peuples amérindiens au Brésil par l'État.

3. Ratifiée par le Brésil en 2002, mais pas encore ratifiée par le Canada ni par l'Australie.

4. Que les gouvernements du Canada et d'Australie refusèrent de ratifier au départ, mais que l'Australie finit par entériner en 2009, et le Canada en 2010

5. Nous utilisons la notion d'indigénisme pour décrire la politique visant l'incorporation des peuples autochtones à l'État national, mais également dans l'acception de Ramos «pour inclure le vaste domaine de l'imaginaire populaire et érudit partagé par la population nationale, dans lequel s'insèrent les multiples facettes de l'autochtone»(Ramos 1998 : 6).

6. Le Territoire du Nord en Australie a une superficie de plus de $1349129 \mathrm{~km}^{2}$ et est la troisième plus grande division fédérale d'Australie. Avec une population de 229675 habitants, il s'agit de la division fédérale la moins peuplée parmi les huit principaux États et territoires d'Australie.

\section{Ouvrages cités}

ALTMAN, Jon, et Melinda HINKSON, 2007 : Coercive Reconciliation: Stabilize, normalize, exit Aboriginal Australia. Arena Publications, Melbourne.

AUSTIN-BROOS, Diane, 2010 : «Quarantining violence: How anthropology does it », in J. Altman et M. Hinkson (dir.), Culture Crisis: Anthropology and politics in Aboriginal Australia: 136-149. UNSW Press, Sydney.

BAINES, Stephen G., 1993 : « O Território dos Waimiri-Atroari e o Indigenismo Empresarial ». Ciências Sociais Hoje : 219-243.

—, 1995: "Primeiras impressões sobre a etnologia indígena na Austrália », in R. Cardoso de Oliveira et G.R. Ruben (dir.), Estilos de Antropologia : 65-119. Editora da UNICAMP, Campinas, SP.

—, 1996 : Etnologia indígena no Canadá: primeiras impressões. DAN/ UnB, Série antropologia 197, Brasília.

—, 2003 : «Organizações Indígenas e legislações indigenistas no Brasil, na Austrália e no Canadá ». Arquivos do Museu Nacional 61(2) : 115-128.

—, 2004 : «Antropologia do desenvolvimento e a questão das sociedades indígenas ». Revista Anthropológicas 15(2) : 29-46.

BECKETT, Jeremy, 1988: «Aboriginality, Citizenship and Nation State ». Aborigines and the State in Australia: Social Analysis 24 : 3-18.

CARDOSO DE OLIVEIRA, Roberto, 1988: Sobre o Pensamento Antropológico. Biblioteca Tempo Universidade $\mathrm{n}^{\circ}$ 83. Tempo Brasileiro, Rio de Janeiro, CNPq, Brasília.

—, 1998 : O trabalho do antropólogo. Paralelo 15, Brasília, Editora UNESP, São Paulo.

DYCK, Noel, 1993: " "Telling it like it is": Some dilemmas of fourth world ethnography and advocacy », in N. Dyck et J.B. Waldram (dir.), Anthropology, Public Policy and Native Peoples in Canada: 192-212. McGill-Queen's University Press, Montréal $\&$ Kingston, London, Buffalo.

DYCK, Noel, et J.B. WALDRAM (dir.), 1993 : Anthropology, Public Policy and native Peoples in Canada. McGill-Queen's University Press, Montréal \& Kingston, London, Buffalo.

GALLOIS, Dominique, 2004 : «Terras ocupadas? Territórios? Territorialidades? » in F. Ricardo (dir.), Terras Indígenas e Unidades de Conservação da Natureza: o desafio das sobreposições : 37-41. Instituto Socioambiental, São Paulo.

HINKSON, Melinda, 2010 : «Introduction: Anthropology and the culture wars », in J. Altman et M. Hinkson (dir.), Culture Crisis: Anthropology and Politics in Aboriginal Australia: 1-13. UNSW Press, Sydney.

KAPFERER, Bruce, 1989 : « Nationalist Ideology and a Comparative Anthropology ». Ethnos 54(3-4) : 161-199.

LUCIANO, Gersem dos Santos, 2006 : O índio brasileiro: o que você precisa saber sobre os povos indígenas no Brasil de hoje. MEC/ SECAD; LACED/Museu Nacional, Coleção Educação Para Todos. Série Vias dos Saberes $n^{\circ} 1$, Brasília. <http://unesdoc. unesco.org/images/0015/001545/154565por.pdf> (consulté le 23 novembre 2014).

MERLAN, Francesca, 2010 : «Child Sexual Abuse: The Intervention Trigger », in J. Altman et M. Hinkson (dir.), Culture in Crisis: Anthropology and Politics in Aboriginal Australia: 116-135. UNSW Press, Sydney.

NORTHERN TERRITORY BOARD OF INQUIRY INTO THE PROTECTION OF ABORIGINAL CHILDREN FROM SEXUAL ABUSE, 2007: Ampe Akelyernemane Meke Mekarle "Little Children are Sacred". Report of the Northern Territory Board of Inquiry into the Protection of Aboriginal Children from Sexual Abuse, Northern Territory Government, Darwin, Australia. $<$ http://www.inquirysaac.nt.gov.au/pdf/bipacsa_final_report. pdf> (consulté le 23 novembre 2014). 
OLIVEIRA, João Pacheco de, 1998 : « Os instrumentos de bordo: expectativas e possibilidades do trabalho do antropólogo em laudos periciais », in J.P. de Oliveira (dir.), Indigenismo e territorialização: poderes, rotinas e saberes coloniais no Brasil contemporâneo : 269-295. Contra Capa Livraria Ltda., Rio de Janeiro.

OLIVEIRA, João Pacheco de, et Marcelo P. IGLESIAS, 2002 : «As demarcações participativas e o fortalecimento das organizações indígenas », in A.C. de S. Lima et M. Barroso-Hoffmann (dir.), Estado e Povos Indígenas: bases para uma nova política indigenista II : 41-68. Contra Capa Livraria/LACED, Rio de Janeiro.

PAINE, Robert (dir.), 1985: Advocacy and Anthropology, First Encounters. Institute of Social and Economic Research, Memorial University of Newfoundland, St. John's.

PEIRANO, Mariza G.S., 1991 [1981] : The Anthropology of Anthropology: The Brazilian case. Série Antropologia, n 110, Departamento de Antropologia, Universidade de Brasília.

PETERSON, Nicolas, 2010 : « Other peoples lives: Secular assimilation, culture and ungovernability, in J. Altman et M. Hinkson (dir.), Culture Crisis: Anthropology and Politics in Aboriginal Australia : 248-258. UNSW Press, Sydney.

POVINELLI, Elizabeth A., 2010 : «Indigenist politics in late liberalism », in J. Altman et M. Hinkson (dir.), Culture Crisis:
Anthropology and Politics in Aboriginal Australia : 17-30. UNSW Press, Sydney.

RAMOS, Alcida Rita, 1990 : «Ethnology Brazilian Style ». Cultural Anthropology 5(4) : 452-472.

—, 1998 : Indigenism: Ethnic politics in Brazil. The University of Wisconsin Press, Madison.

RITTER, David, 2009: The Native Title Market. University of Western Australia Press, Crawley, Western Australia.

SILVA, Cristhian Teófilo da, 2005 : Cativando Maira: a sobrevivência avá-canoeiro no alto rio Tocantins. Annablume, São Paulo.

SUTTON, Peter, 2001 : "The Politics of Suffering: Indigenous policy in Australia since the 1970s». Anthropological Forum 11(2) : 125-172.

—, 2009 : The Politics of Suffering: Indigenous Australia and the end of the liberal consensus. Melbourne University Press, Carleton, Victoria.

WALDRAM, James B., 1993 : « Some limits to advocacy anthropology in the Native Canadian context », in N. Dyck et J.B. Waldram (dir.), Anthropology, Public Policy and Native Peoples in Canada : 293-310. McGill-Queen's University Press, Montréal $\&$ Kingston, London, Buffalo.

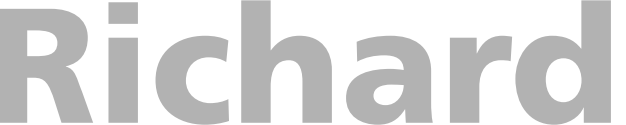

\section{Clash}

Colombins d'argile naturelle, barbotine et teinture, gravé, 2013

Hauteur: 10 po ; diamètre : 15 po

(Photo de I'artiste)
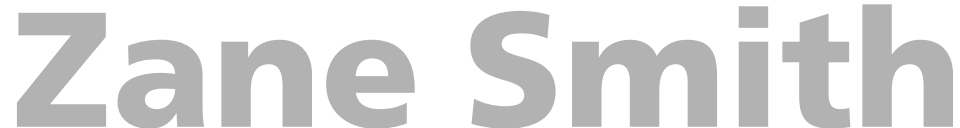

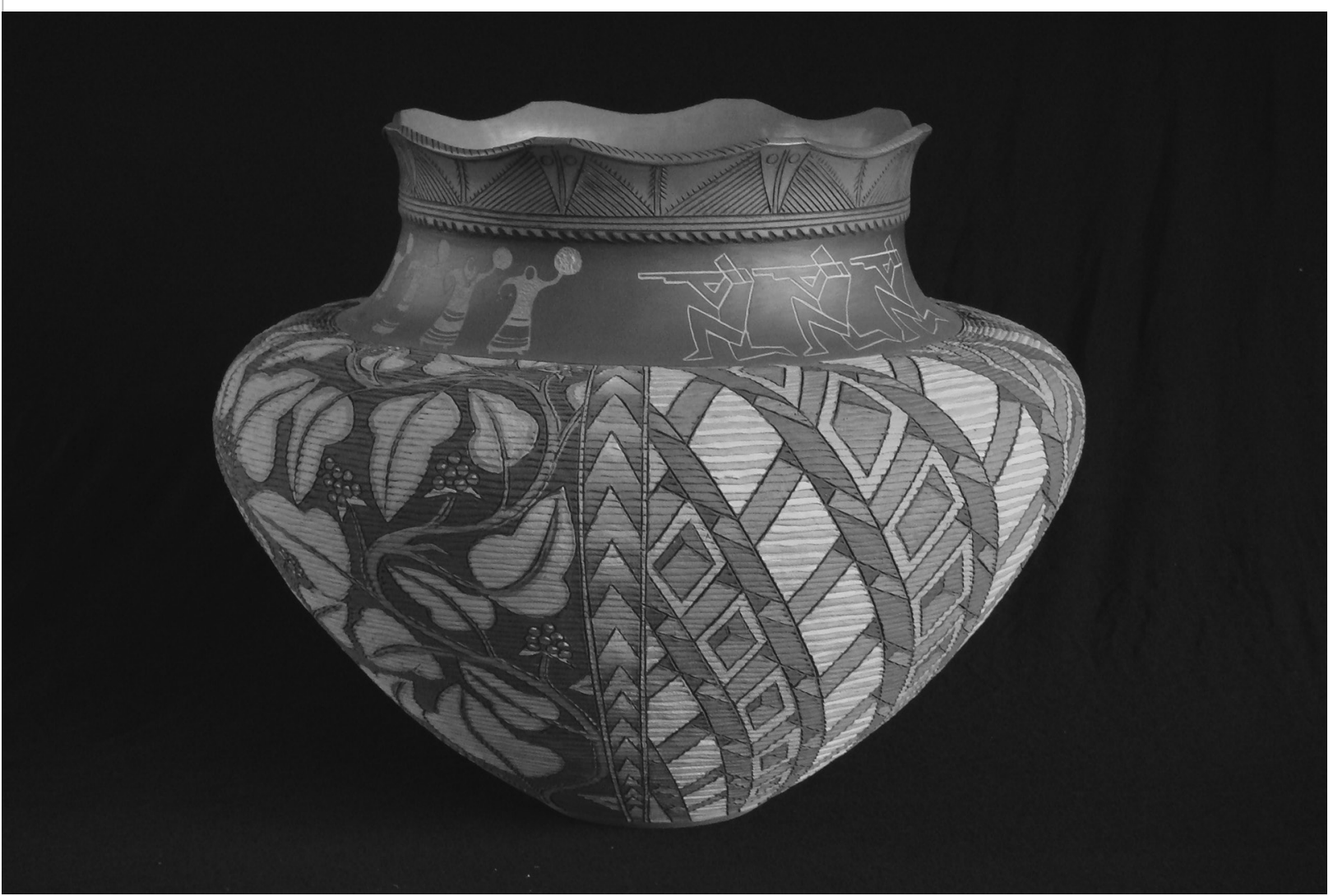

